\title{
Tsafon
}

Revue d'études juives du Nord

$78 \mid 2019$

L'antijudaïsme dans l'Antiquité

\section{La « maladie » du judaïsme chez Jean Chrysostome}

Pierluigi Lanfranchi

\section{(2) OpenEdition}

Journals

Édition électronique

URL : https://journals.openedition.org/tsafon/2307

DOI : $10.4000 /$ tsafon.2307

ISSN : 2609-6420

\section{Éditeur}

Association Jean-Marie Delmaire

\section{Édition imprimée}

Date de publication : 1 décembre 2019

Pagination : 47-64

ISSN : 1149-6630

\section{Référence électronique}

Pierluigi Lanfranchi, « La « maladie » du judaïsme chez Jean Chrysostome », Tsafon [En ligne], 78|

2019, mis en ligne le 01 décembre 2019, consulté le 23 juin 2021. URL : http://

journals.openedition.org/tsafon/2307 ; DOI : https://doi.org/10.4000/tsafon.2307 


\title{
La « maladie » du judaïsme chez Jean Chrysostome
}

Pierluigi Lanfranchi *

Dans son essai Illness as Metaphor, Susan Sontag a décrit le processus de métaphorisation de la maladie en ces termes:

\begin{abstract}
Il n'y a rien de plus punitif que de donner une signification à une maladie - cette signification étant invariablement moraliste. Toute maladie importante dont la cause est obscure et pour laquelle le traitement est inefficace, a tendance à être remplie de significations. Tout d'abord, les choses qui suscitent la crainte la plus profonde (décadence, décomposition, souillure, anomie, faiblesse) sont identifiées avec la maladie. La maladie elle-même devient une métaphore. Ensuite, au nom de la maladie (c'est-à-dire, en utilisant celle-ci comme métaphore), l'horreur est imposée à d'autres objets. La maladie devient un attribut. On dit que quelque chose est comme une maladie pour signifier qu'elle est dégoûtante ou répugnante. ${ }^{1}$
\end{abstract}

La lèpre au Moyen Âge et le cancer de nos jours fonctionnent comme des catalyseurs des sentiments concernant le mal et deviennent des «textes sociaux» dans lesquels le mal se rend visible. Le but polémique de l'écrivaine américaine, elle-même atteinte par un cancer à l'époque de la rédaction de son essai, était de libérer la maladie de ses

\footnotetext{
*Université d'Aix-Marseille.

${ }^{1}$ S. Sontag, Illness as Methaphor, New York, Farrar, Straus and Giroux, 1978, p. 58 : "Nothing is more punitive than to give a disease a meaning - that meaning being invariably a moralistic one. Any important disease whose causality is murky, and for which treatment is ineffectual, tends to be awash in significance. First, the subjects of deepest dread (corruption, decay, pollution, anomie, weakness) are identified with the disease. The disease itself becomes a metaphor. Then, in the name of the disease (that is, using it as a metaphor), that horror is imposed on other things. The disease becomes adjectival. Something is said to be disease like, meaning that it is disgusting or ugly ».
} 
significations symboliques, de résister à la pensée métaphorique, qui était pour elle la façon la plus saine de vivre la maladie (« the healthiest way to be ill »). Sontag voulait qu'on ne considère la maladie que comme maladie et non pas comme un symbole renvoyant à autre chose, fût-il le péché, le désordre moral, la punition divine, etc. Il s'agit d'une aspiration légitime mais difficile, voire impossible, à réaliser. L'homme est par nature un animal symbolique et l'imaginaire lié à la maladie a été exploité depuis toujours pour caractériser ce qui suscite la crainte et la répulsion. Sontag avoue avoir utilisé elle-même le potentiel métaphorique de la maladie lorsqu'elle a écrit, en proie au désespoir face à la guerre des États-Unis contre le Vietnam : «the white race is the cancer of human history $\gg$.

Étant particulièrement puissante et évocatrice, la métaphore de la maladie a été employée dans des contextes de polémique et de conflit religieux à toutes les époques. L'antijudaïsme chrétien et l'antisémitisme moderne ne font pas exception. Pour l'écrivain antisémite autrichien Arthur Trebitsch, le morbus judaicus amené par les Ostjuden était le poison qu'il fallait expulser pour qu'il n'infecte pas l'esprit allemand ${ }^{2}$. $\mathrm{Au}$ Moyen Âge, les auteurs chrétiens ont associé très souvent les Juifs aux maladies contagieuses, telle la lèpre ${ }^{3}$. Mais cette association entre les Juifs et la maladie est beaucoup plus ancienne. Dans cet article, je concentrerai mon attention sur un chapitre de cette longue histoire et j'analyserai la façon dont Jean Chrysostome, dans ses sermons, a décrit les Juifs comme malades et le judaïsme comme maladie.

\section{L'Église malade}

$\mathrm{Au}$ début de son premier sermon contre les Juifs, vraisemblablement prononcé à Antioche à l'automne de l'année $386^{4}$, Chrysostome affirme avoir été obligé d'interrompre la série des homélies consacrées aux anoméens et à leur hérésie, pour aborder un sujet

\footnotetext{
${ }^{2}$ A. Trebitsch, Geist und Judentum : eine grundlegende Untersuchung, Wien, Strache, 1919. Sur la figure de Trebitsch, cf. S.E. Aschheim, Brothers and Strangers. The East European Jew in German and German Jewish Consciousness, 1800-1923, Madison, University of Wisconsin Press, 1982, p. 226.

${ }^{3}$ Voir I.M. Resnick, Marks of Distinction. Christian Perception of the Jews in the High Middle Ages, Washington DC, Catholic University of America Press, 2012.

${ }^{4} \mathrm{Au}$ sujet de la chronologie des sermons contre les Juifs de Chrysostome, voir W. Pradels, R. Brändle and M. Heimgartner, «The Sequence and Dating of the Series ofJohn Chrysostom's Eight Discourses Adversus Iudaeos », Zeitschrift für antikes Christentum 6, 2002, p. 90-116.
} 
beaucoup plus urgent et pressant: «Une autre maladie plus grave demande notre langue pour être soignée, une maladie qui s'est développée dans le corps de l'Église. Il faut d'abord arracher celle-ci et ensuite penser à ceux de l'extérieur, d'abord soigner les nôtres et ensuite s'occuper des étrangers $»^{5}$. De quelle maladie s'agit-il exactement ? Sa nature est précisée aussitôt après :

Quelle est cette maladie? Les fêtes des Juifs misérables et malheureux vont arriver nombreuses et en rapide succession, les trompettes, les tentes, les jeûnes ; et beaucoup de ceux qui se rangent avec nous et affirment penser comme nous, les uns les fréquentent juste pour voir, les autres y participent et s'associent aux jeûnes. Et c'est ce comportement mauvais que je veux expulser de l'Église maintenant. En effet, les discours contre les anoméens peuvent attendre une autre occasion sans qu'il n'y ait aucun dommage à cause de ce retard. Mais ceux qui sont malades de judaïsme, si nous ne les soignons pas maintenant que les fêtes des Juifs approchent et sont aux portes, je crains que certains à cause d'une fâcheuse habitude et d'une grande ignorance ne s'associent à leur transgression et que par la suite nos discours les concernant ne soient inutiles. En effet, s'ils n'entendent rien aujourd'hui et jeûnent avec eux, après avoir commis le péché, ensuite nous administrerons en vain le médicament. Pour cette raison je me presse de prévenir. Ainsi font également les médecins; ils affrontent d'abord les maladies urgentes et plus aiguës. ${ }^{6}$

L'hérésie anoméenne ainsi que ce nouveau danger sont qualifiés de «maladie» $(v o ́ \sigma \eta \mu \alpha)$, mais tandis que la première frappe «ceux de l'extérieur » (oi そ̌ $\xi \omega \theta \varepsilon v)$, à savoir les hérétiques, cette deuxième maladie menace directement les membres de la communauté de Chrysostome. Tous les éléments de la métaphore sont déjà en place : l'Église est représentée comme un «corps» $(\sigma \tilde{\omega} \mu \alpha)$ dans lequel la maladie s'est implantée, le prêtre est le médecin capable de la soigner grâce au pouvoir thérapeutique de sa parole. Dans la sixième des homélies De poenitentia, Chrysostome exprime la même idée :

Ces paroles simples que je viens de lire [il se réfère à la citation de Mt 5, 28] sont suffisantes pour vous purifier de la putréfaction du péché. Mais reconnaissez-le : nous purifions les plaies et il est nécessaire que celui qui purifie les plaies applique des médicaments qui brûlent; et plus vous supportez ces paroles, plus le poison est évacué. ${ }^{7}$

\footnotetext{
${ }^{5}$ Patrologia graeca (désormais PG) 48, 844. Sauf indication contraire, toutes les traductions sont de l'auteur.

${ }^{6} \mathrm{PG} 48,845$.

${ }^{7}$ De poenitentia 6,3 ; PG 49, 317.
} 
La logothérapie pratiquée par Chrysostome sur ses fidèles se fonde sur une longue tradition philosophique et médicale ${ }^{8}$. En effet, comme l'a montré Christopher Gill, les traités philosophiques de la tradition aussi bien platonicienne et aristotélicienne que stoïcienne étaient conçus comme une médecine psychologique préventive pour le contrôle des émotions, ce qu'en termes modernes on pourrait appeler "life-style management $\gg{ }^{9}$. L'analogie entre philosophie et médecine avait déjà été établie par Platon, mais elle devient un topos surtout à l'époque hellénistique dans les écoles stoïcienne et épicurienne. Chrysostome partage avec ces traditions philosophiques l'idée selon laquelle la souffrance humaine est causée par des croyances erronées et qu'il faut changer ces croyances pour prévenir la souffrance. Bien évidemment, dans la perspective de Chrysostome, les « souffrances » $\pi \alpha ́ \theta \eta$ ne sont pas de simples désordres émotionnels, mais des péchés et le but de sa

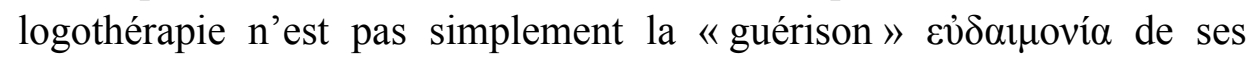
auditeurs, mais le salut de leurs âmes.

À côté de la tradition philosophique grecque, Chrysostome suit aussi la tradition chrétienne du Christ médecin, qui se développe surtout à partir $\mathrm{du} \mathrm{II}^{\mathrm{e}}$ siècle pour concurrencer la popularité des dieux guérisseurs des religions traditionnelles ${ }^{10}$. Jésus agit comme un médecin lorsqu'il opère ses guérisons, par exemple dans le cas du serviteur du centurion (Contra Anomoeos 11, 2 ; PG 48, 799), mais aussi en tant que médecin des âmes (De continentia, p. 581 éd. Haidacher). Pour Chrysostome non seulement Jésus mais aussi les apôtres sont des médecins, dont l'exemple peut être utilisé pour soigner ceux qui judaïsent, comme le dit Chrysostome dans le premier discours contre les Juifs : « En effet nous ne parlons pas maintenant pour faire de l'exhibition ni pour les applaudissements, mais pour soigner vos âmes. En effet, quel discours nous reste-t-il encore, si, malgré de tels médecins (c'est-à-dire les apôtres), certains sont encore malades ? ${ }^{11}$.

\footnotetext{
${ }^{8}$ Voir W. Mayer, «John Chrysostom. Moral Philosopher and Physician of the Soul» dans D. Costache - M. Baghos (eds.), John Chrysostom. Past, Present, Future, Sydney, AIOCS Press, 2017, p. 193-216.

${ }^{9}$ C. Gill, «Philosophical Therapy as Preventive Psychological Medicine», dans W.V. Harris (ed.), Mental Disorder in the Classical World, Leiden, Brill, 2013, p. 339-360.

${ }^{10}$ Pour le thème du Christ médecin dans la littérature chrétienne ancienne, voir l'étude classique de A. von Harnack, Die Mission und Ausbreitung des Christentums in den ersted drei Jahrhunderten, Leipzig, Hinrichs, 1924, vol. 1, p. 129-150. Voir aussi R. Arbesmann, "The Concept of 'Christus Medicus' in St. Augustin », Traditio 10, 1954, p. $1-28$.

${ }^{11}$ PG 48, 849.
} 
Chaque fidèle, et notamment le leader de la communauté, doit imiter le Christ en se faisant lui-même médecin des âmes :

Quand donc tu vois un ennemi de la vérité, soigne-le, occupe-toi de lui, ramènele à la vertu, en lui montrant une vie excellente, en administrant un discours irréprochable, en offrant support et soin, en mettant en place toute sorte de moyen pour sa correction, en imitant les meilleurs médecins. En effet, ceux-ci ne soignent pas par une seule méthode, mais lorsqu'ils voient que la blessure ne répond pas au premier médicament, ils en ajoutent un autre, et après celui-ci un autre encore. Et tantôt ils coupent, tantôt ils lient. Et toi donc étant un médecin des âmes, mets en place toute sorte de thérapie selon les lois du Christ, afin d'obtenir la récompense pour ton propre salut et pour avoir été utile aux autres, en faisant tout pour la gloire de Dieu et étant toi même glorifié par celle-ci. ${ }^{12}$

La logothérapie de Chrysostome est essentiellement une pédagogie par laquelle le prédicateur essaie de persuader sa communauté à poursuivre une vie ascétique en accord avec la volonté du Dieu chrétien, une vie ascétique qui seule peut garantir le salut. Ce faisant, Chrysostome propose un modèle idéal du chrétien et de la communauté chrétienne auquel le fidèle est appelé à se conformer. Mais, comme l'a souligné J.H.W.G. Liebeschuez, la construction d'une identité chrétienne est le résultat, non pas le but premier de la prédication de Chrysostome :

Le but de Chrysostome et de ses collègues était beaucoup plus vaste et leur objectif était beaucoup plus grandiose que la création d'une nouvelle identité. Ils croyaient connaître le plan de Dieu pour la race humaine et ils passèrent leur vie en essayant de le mettre en œuvre. Chrysostome demandait à sa congrégation ce qu'il pensait être la mise en œuvre de la religion chrétienne telle qu'elle avait été fondée dans la Bible et s'était développée et étendue dans les traditions de l’Église. Pour réaliser cela, il fallait à la fois refaçonner la conduite humaine et renforcer et diffuser l'Église. ${ }^{13}$

Le renforcement et l'expansion de l'Église passaient par la fixation de limites externes infranchissables qui devaient préserver la

\footnotetext{
${ }^{12}$ In Mattheum, homélie 29, 3 ; PG 57, 362.

${ }^{13}$ J.H.W.G. Liebeschuez, Ambrose and John Chrysostom. Clerics between Desert and Empire, Oxford, Oxford University Press, 2011, p. 191. «The objective of Chrysostom, and his colleagues, was much wider and their purpose was much grander than the creation of a new identity. They believed that they knew God's plan for the human race, and they spent their lives trying to implement it. What Chrysostom asked of his congregation was what he thought was full implementation of the Christian religion as founded on the Bible and expanded and developed in the traditions of the Church. To achieve this required both reshaping human behaviour, and strengthening and expanding the Church ».
} 
communauté des fidèles de toute contamination avec les éléments allogènes perturbateurs, susceptibles de brouiller la frontière entre ce qui est chrétien et ce qui ne l'est pas. La menace que constitue le judaïsme est particulièrement dangereuse à cause de l'attraction que la religion juive et ses pratiques exerçaient sur les chrétiens d'Antioche. Par sa prédication contre les Juifs à l'intention des chrétiens judaïsants, Chrysostome se propose d'établir un véritable cordon sanitaire pour séparer les deux communautés. C'est bien dans ce cadre qu'il faut comprendre la métaphore de la « maladie » du judaïsme.

\section{La « maladie » du judaïsme}

Comme nous venons de le voir, la maladie du judaïsme est plus grave et plus aiguë que celle de l'hérésie anoméenne et demande donc un traitement prioritaire. Dans les passages du premier discours contre les Juifs que j'ai cités, les images et les termes médicaux employés par Chrysostome sont significatifs. Considérons, par exemple, le verbe « expulser» $\dot{\alpha} \pi \varepsilon \lambda \alpha ́ \sigma \alpha 1$ («Et c'est ce comportement mauvais que je veux expulser de l'Église maintenant»). 'A $\pi \varepsilon \lambda \alpha v ́ v \omega$ revient souvent dans les traités de médecine. Ainsi dans ses Collectiones mediacae, le médecin Oribase, contemporain de Chrysostome, l'utilise au sujet du pouvoir des clystères d'aloès et de figues d'expulser les muqueuses intestinales nocives $(8,26,7)$. Mais on le retrouve également en dehors des textes techniques, par exemple dans les Homélies pseudo-clémentines, où on lit à propos des pouvoirs thaumaturgiques de Jésus : « Il fait en sorte que les sourds entendent, que les aveugles voient, il redresse les courbés et les

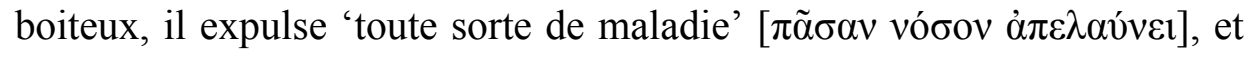
fait fuir toute sorte de démons. Et les lépreux, si seulement le voient de loin, ils s'en vont soignés par lui » $(1,6)$. Nous verrons que le rapport entre maladie et démons joue un rôle fondamental dans l'univers mental de Chrysostome et de son auditoire.

La nécessité d'éradiquer le mal, que Chrysostome identifie avec une opinion erronée ou une croyance fausse, est répétée un peu plus loin dans le premier sermon Adversus Judaeos: «Je sais que beaucoup respectent les Juifs, et pensent que leur mode de vie est pieux. Pour cette raison je me presse d'éradiquer cette opinion pernicieuse $»^{14}$. Ici la métaphore médicale se double de la métaphore végétale, que

\footnotetext{
${ }^{14} \mathrm{PG} 48,847$.
} 
Chrysostome utilise très souvent: pour vaincre la maladie, il faut arracher sa racine ${ }^{15}$.

Le motif de la maladie revient au début du troisième discours, qui ne faisait pas partie de la série des sermons prononcés à l'occasion des fêtes juives de l'automne, mais qui doit être situé peu avant la Pâque juive de l'année 387. Chrysostome ne précise jamais à quel type de maladie le judaïsme doit être assimilé. Néanmoins, il est clair qu'il s'agit pour lui d'une épidémie. Même si après les deux premiers sermons contre les Juifs, le nombre des fidèles atteints par cette maladie a diminué, cela ne dispense pas de la nécessité de les soigner : «Et je sais que, par la grâce de Dieu, le plus grand nombre de ce troupeau s'est libéré de l'infirmité et que la maladie s'est propagée parmi peu de gens ; néanmoins il ne faut pas pour cette raison négliger la thérapie ${ }^{16}$. Donc il faut soigner jusqu'au dernier chrétien judaïsant qui pourrait répandre le mal : «Ne dis pas qu'il ne s'agit que d'une seule personne, car une seule personne, si on la néglige, répandra le mal parmi les autres $»^{17}$. Ces idées ne sont pas limitées aux huit sermons réunis sous les titres Adversus Judaeos. Dans l'une des homélies consacrées à l'exégèse d'Isaïe, Chrysostome interprète les pestiférés qui descendront dans l'Hadès, dont il est question dans Is 5, 14, comme une référence à ceux qui judaïsent : « Il les appelle pestiférés car non seulement ils font du mal à eux-mêmes, mais ils transmettent la maladie aux autres. En effet la peste est de telle nature : lorsqu'elle se sépare d'un corps, elle s'ouvre un chemin et se répand dans le reste de la masse ${ }^{18}$.

En s'adressant ainsi à son auditoire, Jean Chrysostome ne fait pas preuve d'originalité. Il se fonde en effet sur une longue tradition qui faisait des Juifs les porteurs d'une maladie contagieuse, non pas au sens métaphorique, mais au sens propre. La première attestation de cette tradition, bien avant l'essor de l'antijudaïsme chrétien, se trouve dans les écrits des auteurs égyptiens de langue grecque cités par Josèphe dans le Contre Apion. Ainsi Manéthon soutient l'opinion, que Josèphe qualifie de mythe et de discours invraisemblable, selon laquelle les Juifs doivent

\footnotetext{
${ }^{15}$ Cf. par ex. De fato et providentia 2, PG 50, 758 : « Si en effet vous êtes innocents, mais vous connaissez certains qui souffrent de cette maladie, versez sur eux ces paroles et beaucoup d'autres pour que le mal soit éliminé à la racine». Parfois la racine indique la cause profonde de la maladie. Cf. In epistulam ad Romanos 10, 1: «Comme les meilleurs médecins recherchent toujours la racine de la maladie, et remontent à la source du mal, ainsi fait le bienheureux Paul » (PG 60, 476).

${ }^{16}$ PG $48,862$.

${ }^{17} \mathrm{PG} 48,862$.

${ }^{18}$ In Isaiam 5, 6.
} 
être identifiés avec une masse d'Égyptiens lépreux et d'hommes condamnés à quitter l'Égypte à cause d'autres maladies (Ap. 1, 229). Toujours selon Manéthon, le sage Aménophis aurait dit à un roi son homonyme qu'il aurait pu voir les dieux s'il avait purifié tout le pays des lépreux et des autres impurs (Ap. 1, 233) que l'auteur identifie avec les Juifs. Ceux-ci sont également présentés comme mutilés (Ap. 1, 256 ; 260) ou simplement malades (Ap. 1, 257 ; 270). Moïse, qui se serait mis à la tête des lépreux, aurait été lui même un prêtre d'Héliopolis chassé à cause de la lèpre (Ap. 1, 279).

John Barclay remarque que dans les citations littérales du texte de Manéthon faites par Josèphe, il n'est jamais question de lèpre ni de maladies d'autre genre, ni de mutilations, mais seulement d'impureté. Il est probable que Josèphe, dans sa paraphrase de Manéthon, ait " médicalisé » les affirmations de l'auteur égyptien pour montrer leur incompatibilité avec la législation biblique concernant la lèpre. Une autre possibilité serait que le mot lèpre ait été effectivement utilisé par Manéthon, mais seulement pour souligner l'aspect religieux de la souillure avec laquelle les maladies de la peau étaient associées ${ }^{19}$. En réalité, pour la mentalité ancienne, on ne peut pas vraiment distinguer entre le domaine médical et le domaine religieux et entre souillure «profane» et souillure rituelle. Le mythe des Juifs lépreux devait circuler non seulement parmi les savants, mais aussi dans la population égyptienne, si on interprète en ce sens un papyrus de l'époque ptolémaïque ( $\mathrm{I}^{\mathrm{er}} \mathrm{s}$. av. n.è.) dans lequel on lit que les Juifs suscitent du dégoût chez les habitants de Memphis ${ }^{20}$. Il faut peut-être mettre en relation le dégoût des Memphites avec la tradition qui faisait des Juifs des lépreux.

L'association entre les Juifs et la lèpre est reprise par l'historien Lysimaque (Ap. 1, 305), par Apion (Ap. 2, 15) et par Tacite (Historice 5, 3 et 4). Elle perdure encore au $\mathrm{IV}^{\mathrm{e}}$ siècle en milieu « païen », comme en témoigne un fragment d'Helladius d'Alexandrie, un grammairien et prêtre de Zeus, qui s'enfuit à Constantinople en 391: «Celui-ci [Helladius] aussi dit n'importe quoi en affirmant que Moïse était appelé

\footnotetext{
${ }^{19}$ J. Barclay, Flavius Josephus, Against Apion, Leiden, Brill, 2006, 135, nt. 815.

${ }^{20}$ Le papyrus a été publié par R. Remondon, "Les antisémites de Memphis », Chronique d'Egypte 35, 1960, p. 244-261. Il s'agit d'une lettre entre probablement deux Juifs. L'expéditeur écrit au destinataire : «Tu sais qu'ils [c'est-à-dire les habitants de Memphis] éprouvent du dégoût $[\beta \delta \varepsilon \lambda v ́ \sigma \sigma o v \tau \alpha 1]$ pour les Juifs ».
} 


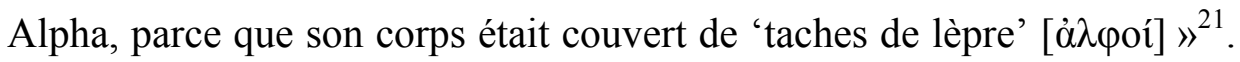
Nous la retrouvons aussi dans la tradition exégétique chrétienne. Selon Origène, Miriam, la sœur de Moïse frappée par la lèpre, serait le symbole de la synagogue (Homélies sur les Nombres VI, 4, 1: «Par surcroît, Marie devient lépreuse. Regarde maintenant ce peuple, vois quelle lèpre le péché a déposé en lui, quel brouillard obscurcit son intelligence, quelle hideur ternit son culte, quelle honte son spectacle $»^{22}$. Athanase dans son commentaire du premier psaume interprète les impies ( $\dot{\alpha} \sigma \varepsilon \beta \varepsilon \tilde{i} \varsigma)$, les pécheurs ( $\dot{\alpha} \mu \alpha \rho \tau \omega \lambda$ oi) et les pestiférés ( $\lambda$ ouooí) du premier verset, comme se référant aux trois groupes du peuple juif, les scribes ( $\gamma \rho \alpha \mu \mu \alpha \tau \varepsilon i \bar{\varsigma})$, les

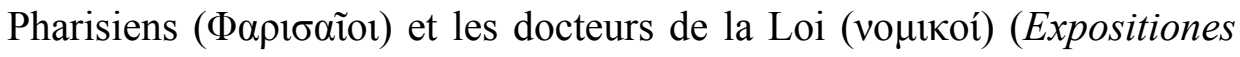
in Psalmos, PG 27, 60).

\section{Maladies curables et maladies incurables}

La métaphore de la maladie ne s'applique pas seulement au judaïsme. Les religions païennes (voir par exemple le passage de Firmicus Maternus cité dans la note 31) ainsi que les hérésies sont considérées par les auteurs chrétiens comme des fléaux. Tout ce qui menace la communauté de l'extérieur peut être assimilé à une maladie contagieuse. Irénée de Lyon affirme que pour convertir les hérétiques il est nécessaire de bien connaître leurs doctrines, de même que pour soigner les malades il faut connaître ce dont ils souffrent ${ }^{23}$. On rappellera que le titre du traité hérésiologique d'Épiphane de Salamine, Panarion, signifie «boîte de médicaments", et qu'Épiphane se réfère à son ouvrage comme à une sorte de médecine basée sur les Écritures saintes et le droit raisonnement pour ceux qui sont malades d'hérésie, et comme à une sorte de prophylaxie pour ceux qui n'ont pas encore contracté la maladie (Panarion 2, 15, 3). Chrysostome suit donc cette tradition lorsqu'il parle de l'hérésie comme d'un vóøฺ $\mu \alpha$.

Mais il y a pour lui une différence essentielle entre la maladie de l'hérésie et celle du judaïsme : la première peut être soignée, tandis que

\footnotetext{
${ }^{21}$ Photius, Bibl., p. 529 b 27. Sur le nom Alpha attribué à Moïse, voir J. Gager, « Moses and Alpha ", Journal of Theological Studies 20, 1969, p. 245-248 et H. Jacobson, «Moses as Alpha », Journal of Theological Studies 50, 1999, p. 626-627. Cf. aussi P. Lanfranchi, «Foetor judaicus. Archéologie d'un préjugé », Pallas 104, 2017, p. 129.

${ }^{22}$ Origène, Homélies sur les Nombres, I: Homélies I-X. Texte latin de W.A. Baehrens (G.C.S.). Nouvelle édition par Louis Doutreleau, d'après l'édition d'André Méhat et les notes de Marcel Borret, (Sources chrétiennes, 415), Paris, Cerf, 1996.

${ }^{23}$ Contra haereses 4, prol. 2.
} 
la deuxième est incurable. Chrysostome le dit dans les discours contre les Juifs, lorsqu'il affirme que le peuple juif ne mérite pas d'indulgence (PG $48,937)$ et, d'une façon encore plus explicite, dans ses Expositiones in Psalmos : «Les Juifs sont irrémédiablement malades $»^{24}$.

Et pourtant Dieu a essayé de soigner les Juifs à plusieurs occasions, notamment lorsqu'il leur a accordé la possibilité de sacrifier des victimes, comme Chrysostome écrit dans le quatrième discours contre les Juifs :

Et comme un médecin, lorsqu'il voit un homme qui a de la fièvre, capricieux et irritable, qui désire des boissons froides et qui menace, s'il ne les obtient pas, de se pendre ou de se jeter des falaises, voulant éviter un mal plus grand, il lui en donne un moindre, car il veut le détourner d'une mort violente. De même a fait Dieu. En effet, après les avoir vus fous, tourmentés, désirant les sacrifices, prêts, s'ils ne les avaient pas obtenus, à passer du côté des idoles, pire non seulement prêts, mais déjà passés, il permit les sacrifices. ${ }^{25}$

Même s'il reconnaît implicitement avoir utilisé un peu trop souvent

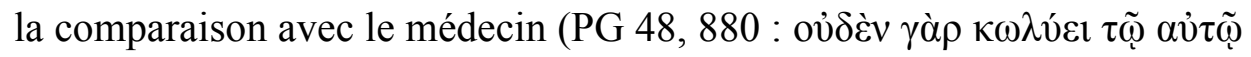

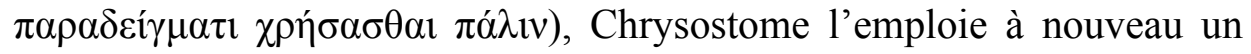
peu plus loin, lorsqu'il s'agit de montrer que Dieu a par la suite enlevé aux Juifs la permission de sacrifier :

Toutefois, après l'avoir octroyé, il ne le permit pas jusqu'à la fin, mais il retira la permission avec une méthode très sage. Et comme ce médecin-là (rien n'empêche d'utiliser à nouveau cet exemple), après avoir cédé au désir du malade, ayant apporté de chez lui une coupe lui ordonne de ne boire des boissons froides que dans celle-ci, et lorsque le malade est persuadé, il ordonne en secret à ceux qui l'administrent de briser la coupe pour le détourner de ce désir sans générer de soupçon (chez le malade) et sans qu'il s'en aperçoive ; ainsi a fait Dieu, en permettant de sacrifier, il ne permit que cela ait lieu dans aucun autre endroit de la terre sauf à Jérusalem, et après qu'ils eurent sacrifié peu de temps, il détruisit la ville afin que, comme le médecin par la destruction de la vaisselle, Dieu aussi les détournât de cette pratique même contre leur gré par l'anéantissement de la ville. ${ }^{26}$

Depuis qu'ils ont tué Jésus, les Juifs ne peuvent plus espérer dans une guérison :

\footnotetext{
${ }^{24}$ Sur le Psaume 8 ; PG 55, 109.

${ }^{25} \mathrm{PG} 48,880$.

${ }^{26}$ Ibid.
} 
Tant que vous avez péché contre vos esclaves, vous avez été pardonnés, même si vous aviez tué et lapidé les prophètes. Mais depuis que vous avez mis vos mains sur le Maître, alors votre plaie est devenue incurable. ${ }^{27}$

Le temps où Dieu se souciait des Juifs comme un médecin prévenant est révolu. Les Juifs, dans leur obstination, ne sont même pas disposés à accepter la miséricorde que Dieu leur avait offerte :

Mais il y a des gens qui n'acceptent pas la miséricorde, car ils sont atteints d'une maladie incurable, comme l'étaient les Juifs ; en effet, la grâce et la miséricorde, si elles sont grâce et miséricorde, ne sauvent que ceux qui le veulent et sont reconnaissants, non pas ceux qui se révoltent et ne veulent pas accepter le don, comme l'ont fait les Juifs. ${ }^{28}$

L'idée selon laquelle on ne peut pas soigner ceux qui n'acceptent pas de se faire soigner est exprimée aussi dans De sacerdotio II, $3^{29}$.

Dans un fragment sur Isaïe qui se trouve dans les chaînes, Chrysostome en commentant le verset «Écoute la parole du Seigneur, Judée toute entière » écrit :

S'ils pèchent irrémédiablement, et s'ils sont atteints d'une maladie incurable, et qu'il n'y a aucune consolation pour leurs actions terribles, pourquoi envoie-t-il à nouveau un médecin, qui ne fera qu'augmenter leur souffrance et qui leur enlèvera toute possibilité d'être pardonnés ? En effet, celui qui écoute souvent et néanmoins il ne se corrige pas, a le plus grand accusateur dans le conseil et l'exhortation souvent répétés. ${ }^{30}$

Dans la partie perdue du deuxième sermon Adversus Judaeos qui a été retrouvée il y a une vingtaine d'années par Wendy Pradels dans le monastère de Leimon sur l'île de Lesbos, Chrysostome compare l'Ancien et le Nouveau Testament à deux médecins :

Et en effet supposons qu'il y ait deux médecins, l'un moins bon, l'autre meilleur, et que le premier, ayant appliqué des médicaments aux blessures, n'arrive pas à libérer une fois pour toutes la personne malade de la douleur qu'elles lui causent, mais lui apporte seulement un léger soulagement. Le docteur plus qualifié, en revanche, une fois arrivé, en levant tous ces médicaments et en lavant

\footnotetext{
${ }^{27}$ PG 55, 111.

${ }^{28}$ Sur le Psaume 5 ; PG 55, 66.

${ }^{29}$ Sur le lexique de la maladie dans le De sacerdotio, voir J. Ángel y Espinós, « La lengua médica en el diálogo Sobre el sacerdotio de Juan Crisóstomo ", Atene e Roma 12, 2018, p. 103-125.

${ }^{30}$ PG 64, 832.
} 
simplement la personne souffrante, est capable de la déclarer purifiée de ses blessures, n'ayant pas de séquelles ni d'éventuelle trace ${ }^{31}$. Supposons qu'ensuite le premier empêche que le patient soit traité par le deuxième. Quel bénéfice peutil apporter par l'application des médicaments qui serait aussi grand que le dommage qu'il a causé en empêchant le patient d'arriver à une guérison immédiate et très rapide ? ? $^{32}$

En d'autres termes, on ne peut pas guérir du judaïsme si on continue à faire confiance aux soins de l'Ancienne Alliance, mais on peut être sauvé si on se met dans les mains du médecin plus compétent, la Nouvelle Alliance, qui régénère avec l'eau du baptême. C'est ainsi que Chrysostome peut terminer son quatrième discours Adversus Judaeos par une prière pour le salut des égarés et pour la conversion des Juifs.

\section{Quelle thérapie pour soigner du judaïsme?}

De la lecture des sermons de Jean Chrysostome il ressort que le caractère incurable de la maladie du judaïsme est lié aux rapports que les Juifs entretiennent avec les démons. Déjà Justin avait suggéré la présence d'un grand nombre de possédés chez les Juifs, que l'apologiste explique par le fait que leurs exorcismes auraient été inefficaces, sauf ceux prononcés au nom de Dieu :

Mais si vous [les Juifs] exorcisez au nom de l'un de vos rois, ou de vos justes, ou de vos prophètes ou patriarches, aucun des démons ne sera soumis. Si toutefois l'un de vous exorcise par le Dieu d'Abraham, le Dieu d'Isaac et le Dieu de Jacob, peut-être il [le démon] sera soumis. Mais désormais, disais-je, vos exorcistes exorcisent grâce à l'art [magique], comme le font les gentils, et ils utilisent des fumigations et enchantements. ${ }^{33}$

Jean Chrysostome va encore plus loin. Pour lui tous les Juifs sont possédés par les démons qui ont pénétré dans leurs âmes. Même s’il ne le

\footnotetext{
${ }^{31}$ Firmicus Maternus utilise une formulation très proche de celle de Chrysostome, au sujet des religions païennes qu'il considère comme des maladies mentales transmissibles, qui peuvent être soignées pas un discours persuasif jusqu'à ce qu'il n'y ait pas de traces de l'ancienne pestilence dans les esprits ainsi purifiés: Vt autem miseros homines ad sanam mentem reuocet plena persuasio, maiore auctoritate opus est, ut curatis ac sanatis mentibus nullum praecedentis pestilentiae uestigium relinquatur (De errore profanarum religionum 28,1 ).

${ }^{32}$ Cf. W. Pradels, R. Brändle, M. Heimgartner, « Das bisher vermisste Textstueck in Johannes Chrysostomus, Adversus Judaeos, Oratio 2 », Zeitschrift für antikes Christentum 5/1, 2001, p. 30.

${ }^{33}$ Dialogue avec Tryphon 85, 3.
} 
dit pas explicitement, la présence des démons dans les âmes des Juifs est favorisée par leur nature irrationnelle. En effet, les Juifs sont souvent qualifiés par Chrysostome de ö $\lambda$ o $\gamma \alpha$, bêtes dépourvues de raison (cf. PG $48,486 ; 853)^{34}$.

Il y a donc une relation très étroite entre la maladie dont souffrent les Juifs et le fait qu'ils sont possédés par les démons. Il suffit de lire ce passage tiré du premier discours contre les Juifs :

\begin{abstract}
Ne vois-tu pas que les démons habitent dans leurs âmes, et ceux d'aujourd'hui sont pires que ceux d'avant. Et ce n'est pas étonnant. En effet, auparavant ils ont été impies contre les prophètes, tandis que maintenant ils ont commis de la violence contre celui-ci, le Seigneur des prophètes. Donc, dis-moi, vous vous réunissez avec des personnes possédées par les démons et qui ont de tels esprits impurs, qui ont grandi dans les égorgements et les meurtres, et vous n'avez pas peur ? Faut-il en effet leur dire bonjour, échanger un discours simple ou plutôt les éviter comme une pestilence commune et comme une pandémie ? ${ }^{35}$
\end{abstract}

Celui qui fréquente les Juifs s'expose donc au risque d'être possédé par les démons. Cela arrive aussi aux chrétiens qui se font soigner par les médecins juifs. Être soigné par les Juifs signifie, selon Chrysostome, demander la santé du corps aux démons et renoncer par conséquent au salut de l'âme :

Si nous sommes malades, il vaut mieux rester dans l'infirmité plutôt que de tomber dans l'impiété pour être délivrés de la maladie; en effet, si un démon nous avait soigné, il nous aurait causé un dommage plus grand qu'un avantage. Car il aurait apporté un avantage au corps, qui va mourir en tout cas peu de temps après et va pourrir, mais il aurait endommagé l'âme immortelle. ${ }^{36}$

Dans ses sermons Adversus Judaeos, Chrysostome se montre moins intéressé par la conversion des Juifs que par l'élimination des pratiques et des tendances judaïsantes à l'intérieur de sa propre communauté. Comme nous l'avons remarqué, la maladie du judaïsme est très clairement présentée comme une maladie contagieuse et transmissible ${ }^{37}$. D'où l'importance d'éteindre tout foyer d'infection :

\footnotetext{
${ }^{34}$ L'idée selon laquelle les démons s'insinuent avec plus de facilité dans les âmes des personnes irrationnelles se trouve chez Athénagore d'Athènes, Supplique au sujet des chrétiens 27.

${ }^{35}$ PG 48,852 .

${ }^{36} \mathrm{PG} \mathrm{48,855.}$

${ }^{37}$ Voir S. Jarco, The Concept of Contagion in Medicine, Literature, and Religion, Malabar FL, Krieger, 2000, p. 30-31.
} 
Si vous voyez l'un de vos frères tomber dans une telle transgression, vous croyez qu'il s'agit du malheur d'un autre, qui ne vous concerne pas, et vous pensez vous justifier face à ceux qui vous adressent des reproches en disant: 'Qu'est-ce que ça peut bien me faire? Je n'ai aucune relation avec celui-ci', en prononçant des mots d'une extrême misanthropie et d'une férocité diabolique. Que dis-tu ? Tu es un être humain et participes de la même nature, ou plutôt, s'il faut parler d'une nature commune, tu as une seule tête, le Christ, et tu oses dire que tu n'as rien en commun avec les parties de ton corps? Comment donc peux-tu reconnaitre que le Christ est la tête de l'Église ? Et en effet, la tête par sa nature unit toutes les parties du corps, les dirige avec précision vers elles-mêmes et les lie ensemble. Si tu n'as rien en commun avec une partie de ton corps, tu n'as rien en commun avec ton frère et tu n'as pas le Christ comme tête. ${ }^{38}$

En évoquant la métaphore paulinienne du corps de l'Église, Chrysostome insiste sur l'idéal de l'unité de la communauté ecclésiale qui a été toujours la plus grande préoccupation de Paul : «Et comme un excellent médecin soigne les blessures et les fractures seulement après avoir fait baisser la fièvre, ainsi a fait Paul : il a d'abord éliminé la discorde, ensuite il a soigné les blessures de chaque partie ${ }^{39}$. En commentant 1 Cor. 12, 12, Chrysostome dit :

Comme en effet, dit-il, notre corps est une seule chose, même s'il se compose de plusieurs membres, ainsi nous aussi nous sommes tous une seule chose dans l'Église, car même si celle-ci se compose de plusieurs membres, néanmoins tous ceux-ci ne font qu'un seul corps. ${ }^{40}$

Et si l'individu doit chasser la maladie de son corps, de même la communauté, formant un seul corps, doit soigner les parties malades.

Chrysostome est persuadé que la meilleure cure est la prévention. C'est ainsi qu'au début du deuxième sermon contre les Juifs, il justifie le fait d'avoir prononcé le premier sermon bien avant le début du jeûne des Juifs sur la base du principe de précaution. Encore une fois c'est par une comparaison médicale qu'il s'adresse à son auditoire :

Ayant pris les devants, il y a dix jours ou plus je vous ai adressé une exhortation pour protéger vos frères. Mais personne ne me reproche d'avoir choisi un moment intempestif pour mon discours, car je l'ai anticipé tant de jours. Et en effet, lorsqu'on soupçonne la présence de la fièvre ou d'une autre maladie, par précaution ils [les médecins] protègent le corps de celui qui va être frappé avec

\footnotetext{
${ }^{38} \mathrm{PG} \mathrm{48,848.}$

${ }^{39} \mathrm{PG} 48,864$.

${ }^{40}$ In Epistulam I ad Corinthios 30, 1 dans PG 61, 250, 47-51.
} 
beaucoup de traitements, en se hâtant, avant qu'il n'éprouve l'attaque, de le soustraire aux maux qui surgissent. Comme nous voyons la maladie très grave qui va se manifester, nous l'avons déclarée dès l'origine avec beaucoup d'avance, pour avoir le traitement avant d'éprouver le mal. Pour cette raison nous n'avons pas prononcé notre exhortation peu de temps avant ces jours-ci, pour que le manque de temps ne vous empêche d'aller à la chasse de vos frères, mais disposant de l'aisance que votre grand nombre vous assure, en toute sécurité vous puissiez rechercher ceux qui souffrent de cette maladie et leur redonner la santé. ${ }^{41}$

L'enseignement que les fidèles reçoivent à l'Église doit d'un côté les immuniser face aux tendances judaïsantes, de l'autre côté leur permettre de reconnaître l'erreur chez leurs frères et de ramener ceux-ci dans le droit chemin : «Je ne dis pas cela seulement à eux, mais aussi à vous qui êtes sains, pour que, si vous en voyez qui sont dans cet état, vous les repériez et rassembliez avec beaucoup de zèle et de douceur, et les conduisiez à leur mère $»^{42}$. Il ne faut pas renoncer, même si les frères judaïsants se montrent récalcitrants et insultent ou frappent les fidèles qui veulent les sauver. Les médecins aussi sont souvent insultés et frappés par leurs patients :

Et en effet, nous supportons les malades même s'ils donnent des coups de pieds et sont violents et nous insultent, ne sommes pas affligés par leurs violences, mais nous désirons voir une seule chose, la santé de celui qui se comporte si mal. Et souvent le malade déchire la veste du médecin ; mais celui-ci ne s'abstient pas pour autant de le soigner. N'est-il donc pas incroyable que ceux qui prennent soin des corps mettent tant de diligence tandis qu'ils sont indifférents si tant d'âmes sont détruites, et qu'on considère qu'il ne se passe rien de grave si les membres de notre corps pourrissent. ${ }^{43}$

Pour ceux qui n'ont pas profité de la médecine préventive de la parole de Chrysostome et qui ont péché en se rendant aux fêtes juives, la thérapie doit être répétée, comme il le dit dans le deuxième discours Adversus Judaeos :

Mais maintenant, après avoir laissé de côté la lecture des discours contre ceux-ci [c'est-à-dire les Juifs], il est temps d'adresser ma critique contre ceux qui sont allés écouter les trompettes. En effet, il n'aurait pas fallu les considérer par la suite dignes d'être mentionnés, car après de telles exhortations et de tels conseils ils ont persisté dans leur folie. Mais puisque je m'attends à les corriger par cette

\footnotetext{
${ }^{41} \mathrm{PG} 48,857$.

${ }^{42} \mathrm{PG} 48,870$.

${ }^{43} \mathrm{PG} \mathrm{48,882.}$
} 
deuxième exhortation et à les convaincre de reconnaître leur folie également quant à leurs comportements précédents, je m'engage avec ferveur dans ces discours à leur intention. Et en effet, je sais que, par la grâce de Dieu, beaucoup de ceux qui avaient l'habitude de faire cela, après mon exhortation, ont abandonné cette mauvaise habitude. Et s'ils n'ont pas été tous persuadés, ils le seront certainement. Car un corps qui a commencé à guérir, faisant des progrès, se débarrassera de toute maladie et atteindra un état de pure santé. ${ }^{44}$

Chrysostome a prévu des cures et des médicaments différents non seulement selon la gravité de la maladie, mais aussi selon le niveau d'initiation du fidèle qui en est atteint :

Si celui qui souffre d'une telle maladie est un catéchumène, qu'il soit éloigné des portes [de l'Église]; s'il est un fidèle ou un initié, qu'il soit exclu de la sainte table. En effet, les péchés ne nécessitent pas tous une exhortation ni un avis, mais il $\mathrm{y}$ en a qui par leur nature sont corrigés par une excision rapide et très nette. Et de même que les blessures plus supportables répondent à des médicaments plus doux, tandis que celles purulentes, incurables et qui consomment le corps, exigent une lame aiguisée et une flamme, ainsi parmi les péchés certains ont besoin d'une exhortation plus longue, d'autres des réfutations concises. $^{45}$

\section{Conclusion}

La métaphore de la maladie sert à Chrysostome pour présenter à ses auditeurs la religion juive comme un danger réel et concret aussi bien pour la vie de chaque chrétien que pour l'ensemble de la communauté, pour l'âme de l'individu et pour le corps de l'Église. Dans le De sacerdotio, Jean a développé l'idée selon laquelle la mission du prêtre est de prendre soin du corps de l'Église :

En effet, selon le bienheureux Paul, l'Église du Christ, c'est le corps du Christ et il faut que celui à qui on le confie s'efforce de le garder en parfait état, de contribuer à sa beauté inégalable, veillant sur tous les points à ce que ni tache, ni ride, ni aucun autre défaut ne souille cette jeunesse et cette beauté $(4,2$, 99$104)^{46}$

\footnotetext{
${ }^{44}$ Le texte se trouve dansW. Pradels, R. Brändle, M. Heimgartner, « Das bisher vermisste Textstueck in Johannes Chrysostomus, Adversus Judaeos, Oratio 2 », Zeitschrift für antikes Christentum 5/1, 2001, p. 44.

${ }^{45}$ PG 48, 862.

${ }^{46}$ Jean Chrysostome, Sur le sacerdoce. Introduction, texte critique, traduction et notes par Anne-Marie Malingrey, (Sources Chrétiennes 272), Cerf, Paris 1980.
} 
Et un peu plus loin : «Ne sais-tu pas que ce corps est exposé à plus de maladies et de dangers que notre chair, qu'il se détériore plus vite et qu'il se remet plus lentement?»(4,3,1-5). Les taches qui souillent l'Église sont là aussi les erreurs des païens, des hérétiques et des Juifs que le prêtre doit savoir réfuter grâce à sa parole et à son éloquence. Dans les sermons Adversus Judaeos ainsi que dans le reste de sa prolifique production homilétique, Chrysostome met en pratique ce qu'il a théorisé dans le traité Sur le sacerdoce.

La maladie du judaïsme est d'autant plus dangereuse qu'elle frappe non pas des gens du dehors, comme c'était le cas pour les anoméens, mais les membres mêmes de la communauté de Chrysostome. L'opposition entre ceux de l'intérieur et ceux de l'extérieur répond au mécanisme fondamental de la stratégie polémique de Chrysostome contre les Juifs, à savoir la dichotomisation entre «nous » et « eux » qui peut se décliner en d'autres oppositions: purs/impurs, spirituels/corporels, sains/malades, etc. Cette dichotomisation sert à tracer une frontière entre les chrétiens et les autres, qu'ils soient Juifs, paiens ou hérétiques.

Parfois on a l'impression que pour Chrysostome le vóøๆ $\mu \alpha$ des Juifs est plus qu'une simple métaphore. Cela est suggéré par l'insistance sur la présence des démons dans les lieux de cultes, voire dans les âmes des Juifs. Pour Chrysostome et pour son public, les démons, loin d'être des créatures fictives, des épouvantails rhétoriques, sont des entités bien réelles, responsables de certaines maladies et de certains péchés et objet d'une répulsion et d'un dégoût profonds. L'efficacité de la logothérapie comme médicament pour les malades et comme prophylaxie pour les sains dépend aussi de la capacité de l'orateur à mobiliser les peurs et les craintes de ses auditeurs, celles de la contagion, de la souillure, de l'impureté, des démons.

$* * * * * * * * * * * * * * * * * *$

\section{Bibliographie}

Ángel y Espinós J., « La lengua médica en el diálogo Sobre el sacerdotio de Juan Crisóstomo », Atene e Roma 12, 2018, p. 103-125.

Arbesmann R., «The Concept of 'Christus Medicus' in St. Augustin », Traditio 10, 1954, p. 1-28. 
Aschheim S.E., Brothers and Strangers. The East European Jew in German and German Jewish Consciousness, 1800-1923, Madison, University of Wisconsin Press, 1982.

Barclay J., Flavius Josephus, Against Apion, Leiden, Brill, 2006.

Gager J., « Moses and Alpha », Journal of Theological Studies 20, 1969, p. 245-248.

Gill C., " Philosophical Therapy as Preventive Psychological Medicine », dans W.V. Harris (ed.), Mental Disorder in the Classical World, Leiden, Brill, 2013, p. 339-360.

Harnack A. von, Die Mission und Ausbreitung des Christentums in den ersted drei Jahrhunderten, Leipzig, Hinrichs, 1924.

Jacobson H., «Moses as Alpha », Journal of Theological Studies 50, 1999, p. 626-627.

Jarco S., The Concept of Contagion in Medicine, Literature, and Religion, Malabar FL, Krieger, 2000.

Lanfranchi P., « Foetor judaicus. Archéologie d'un préjugé », Pallas 104, 2017, p. 119-133.

Liebeschuez J.H.W.G., Ambrose and John Chrysostom. Clerics between Desert and Empire, Oxford, Oxford University Press, 2011.

Mayer W., "John Chrysostom. Moral Philosopher and Physician of the Soul » dans D. Costache - M. Baghos (eds.), John Chrysostom. Past, Present, Future, Sydney, AIOCS Press, 2017, p. 193-216.

Pradels W., Brändle R. and Heimgartner M., « Das bisher vermisste Textstueck in Johannes Chrysostomus, Adversus Judaeos, Oratio 2 », Zeitschrift für antikes Christentum 5/1, 2001, p. 23-49.

Pradels W., Brändle R. and M. Heimgartner, « The Sequence and Dating of the Series ofJohn Chrysostom's Eight Discourses Adversus Iudaeos », Zeitschrift für antikes Christentum 6, 2002, p. 90-116.

Remondon R., «Les antisémites de Memphis », Chronique d'Égypte 35, 1960, p. 244-261.

Sontag S., Illness as Methaphor, New York, Farrar, Straus and Giroux, 1978.

Trebitsch A., Geist und Judentum : eine grundlegende Untersuchung, Strache, Wien, 1919. 